\title{
Pyknodysostosis: report of a rare case with review of literature
}

\author{
Kiran Kumar Kotagudda Ramaiah, Giju Baby George, Sheeba Padiyath, Rupak Sethuraman, Babu Cherian* \\ Department of Oral Medicine and Radiology, Mar Baselios Dental College, Kothamangalam, India \\ *Department of Prosthodontics, Mar Baselios Dental College, Kothamangalam, India
}

\section{ABSTRACT}

\begin{abstract}
Pyknodysostosis is a rare autosomal recessive disorder characterized by the post natal onset of short limbs, short stature, and generalized hyperostosis along with acro-osteolysis with sclerosis of the terminal phalanges, a feature that is considered essentially pathognomonic. Other features include persistence of fontanelles, delayed closure of sutures, wormian bones, absence of frontal sinuses, and obtuse mandibular gonial angle with relative mandibular prognathism. We report a case of 17-year-old girl who presented with a chief complaint of retention of deciduous teeth. General physical examination demonstrated short stature, frontal and parietal bossing, depressed nasal bridge, beaked nose, hypoplastic midface, wrinkled skin over the finger tips, and nail abnormalities. Radiographs showed multiple impacted permanent and supernumerary teeth, hypoplastic paranasal sinuses with acro-osteolysis of terminal phalanges, and open fontanelles, and sutures along with wormian bones in the lambdoidal region. (Imaging
\end{abstract}

Sci Dent 2011; 41 : 177-81)

KEY WORDS : Pyknodysostosis; Craniofacial Abnormalities; Dysostoses; Acro-Osteolysis

Pyknodysostosis, which was first described in 1962 by Maroteaux and Lamy, is a rare autosomal recessive disorder of osteoclast dysfunction causing osteosclerosis. The name derives from the Greek "pyknos" meaning "dens". These authors speculated that the famous French painter, Toulouse-Lautrec (1864-1901), may have suffered from this disease. ${ }^{1}$ The disease shows equal sex distribution with high parental consanguinity, having an incidence of 1.7 per 1 million births. ${ }^{2,3}$ A common finding in this disorder is a greater tendency for fractures, especially of the long bones. Spontaneous fractures of the mandible during mastication are uncommon and are usually due to trauma, exodontias, and post-osteomyelitis. ${ }^{4}$

General features of pyknodysostosis include short stature under $150 \mathrm{~cm}$, generalized diffuse osteosclerosis with a tendency for fracture after minimal trauma, hypoplastic clavicles, as well as acro-osteolysis with sclerosis of the

Received July 6, 2011; Revised July 22, 2011; Accepted October 1, 2011 Correspondence to: Dr. Kiran Kumar Kotagudda Ramaiah

Department of Oral Medicine and Radiology, Mar Baselios Dental College, Thankalam, Kothamangalam, Pin-686691, Ernakulam District, Kerala, India

Tel) 91-953-9631813, Fax) 91-485-2828745, E-mail) krksmile@ gmail.com terminal phalanges which is an essential pathognomonic feature. Other features include wrinkled skin, finger and nail abnormalities, kyphosis and scoliosis, history of repeated chest infections, and sleep apnea. The intellectual and sexual development is usually normal in the patients. ${ }^{5,6}$ Cranial and maxillofacial features include frontoparietal bossing, thick calvaria, open fontanelles and sutures, hypoplastic paranasal sinuses, wormian bones in the lambdoidal region, relative proptosis, beaked nose, hypoplastic midface, and obtuse mandibular gonial angle, often with relative prognathism. ${ }^{5}$

\section{Case Report}

A 17-year-old girl reported to the Department of Oral Medicine with a chief complaint of retention of deciduous teeth. On general physical examination (Fig. 1), she demonstrated short stature, frontal and parietal bossing, depressed nasal bridge, beaked nose, hypoplastic midface, obtuse mandibular gonial angle with relative prognathism, wrinkled skin over the finger tips, and nail abnormalities. Intraoral examination revealed deep midline antero-posterior 

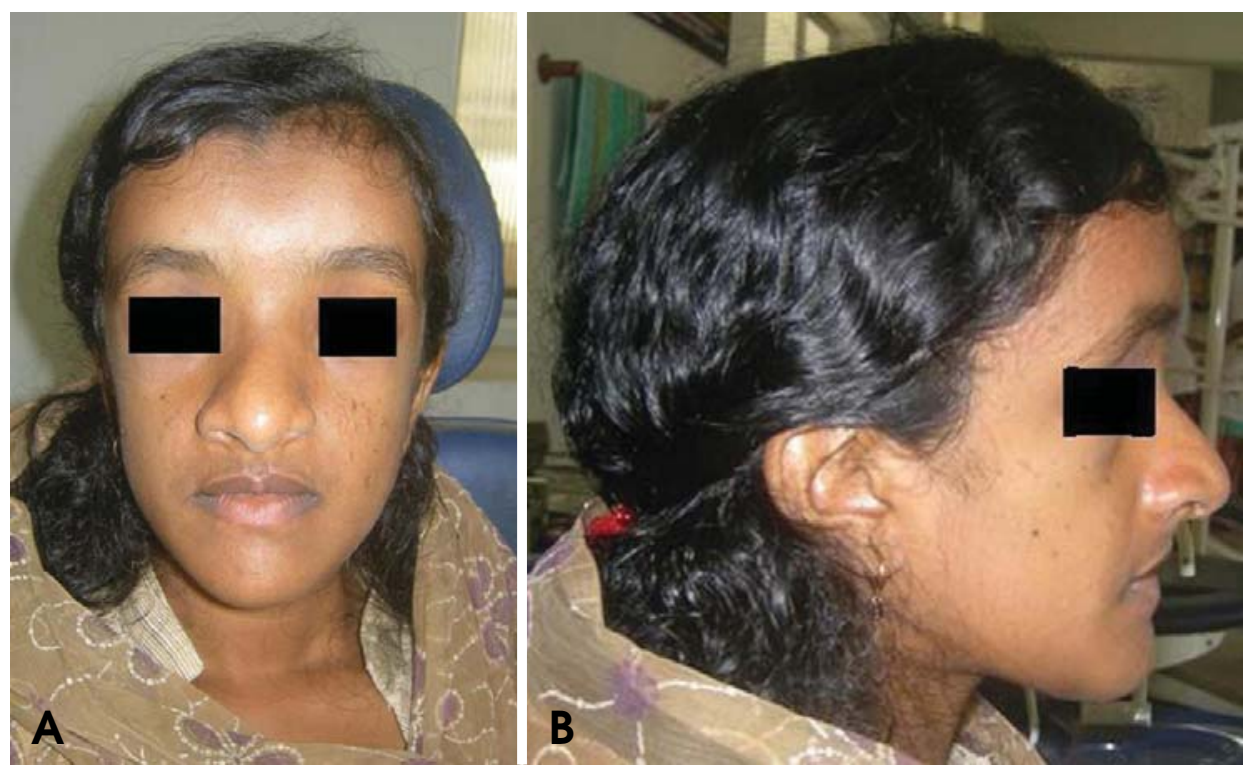

Fig. 1. A and B. Clinical photographs show frontal bossing, beaked nose and midfacial hypoplasia.

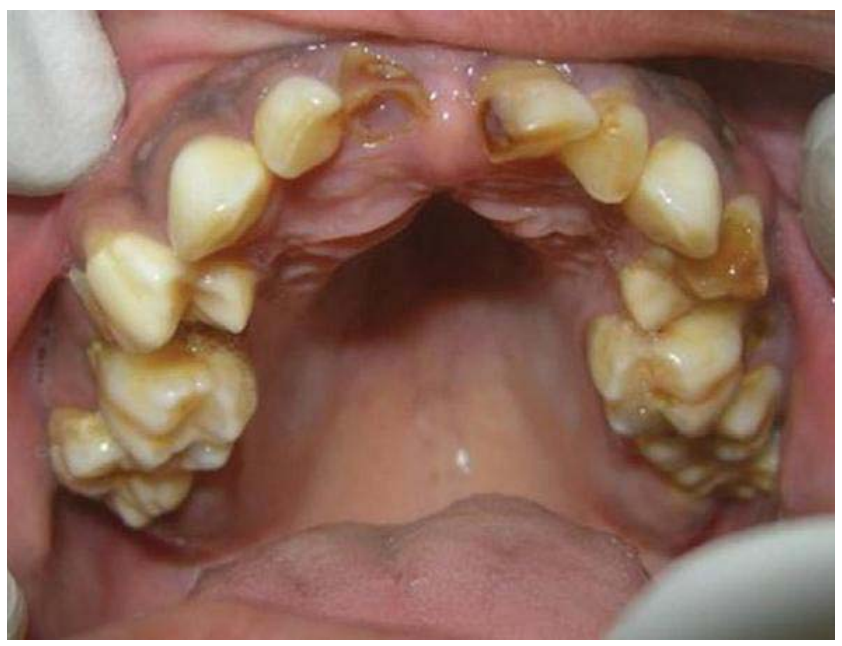

Fig. 2. Intraoral photograph shows $\mathrm{V}$-shaped high arched palate with midline groove and multiple retained deciduous teeth.

ridge of the palate, high arched palate, multiple retained deciduous carious teeth, crowding, and enamel hypoplasia (Fig. 2). Based on the above findings, the differential diagnoses include osteopetrosis, pyknodysostosis, cleidocranial dysplasia, and idiopathic acro-osteolysis.

Panoramic radiograph showed multiple impacted permanent and supernumerary teeth with narrow left ramus, condyle, and condylar notch (Fig. 3A). Lateral cephalogram showed hypoplastic paranasal sinuses with relative mandibular prognathism (Fig. 3B). Handwrist radiograph showed acro-osteolysis of terminal phalanges (Fig. 3C). Three dimensional CT image revealed open fontanelles and sutures, wormian bones in the lambdoidal region (Fig. 4).
Based on the above investigative reports, the final diagnosis of pyknodysostosis was made.

\section{Discussion}

Pyknodysostosis is an autosomal recessive disorder of bone, causing osteoclast dysfunction resulting in osteosclerosis. The sclerosing activity of pyknodysostosis is due to a genetic defect located on chromosome 1q21. This anomaly consists of mutations that produce mutational changes in a lysosomal cystine protease, cathepsin $\mathrm{K}$ (CTSK), the expression of which is reduced in the osteoclasts. This protease is responsible for degrading Type 1 collagen that constitutes $95 \%$ of the organic bone matrix. The affected bones are abnormally dense and brittle as a result of insufficient resorption. ${ }^{7,8}$

In our case study, the general features present were short stature, wrinkled skin, finger and nail abnormalities as well as acro-osteolysis with sclerosis of the terminal phalanges which is an essential pathognomonic feature. The same findings have been reported by Hunt et $\mathrm{al}^{5}$ and Schmitz et al. ${ }^{6}$ Cranial and maxillofacial features reported in our case included frontoparietal bossing, open fontanelles and sutures, hypoplastic paranasal sinuses, wormian bones in the lambdoidal region, beaked nose, hypoplastic midface, and obtuse mandibular gonial angle, often with relative prognathism. These findings correlate with those reported by Hunt et al ${ }^{5}$ and Landa et al. ${ }^{9}$

According to some studies, intraoral features such as persistence of deciduous teeth with premature or delayed eruption of permanent teeth leading to crowding, enamel 

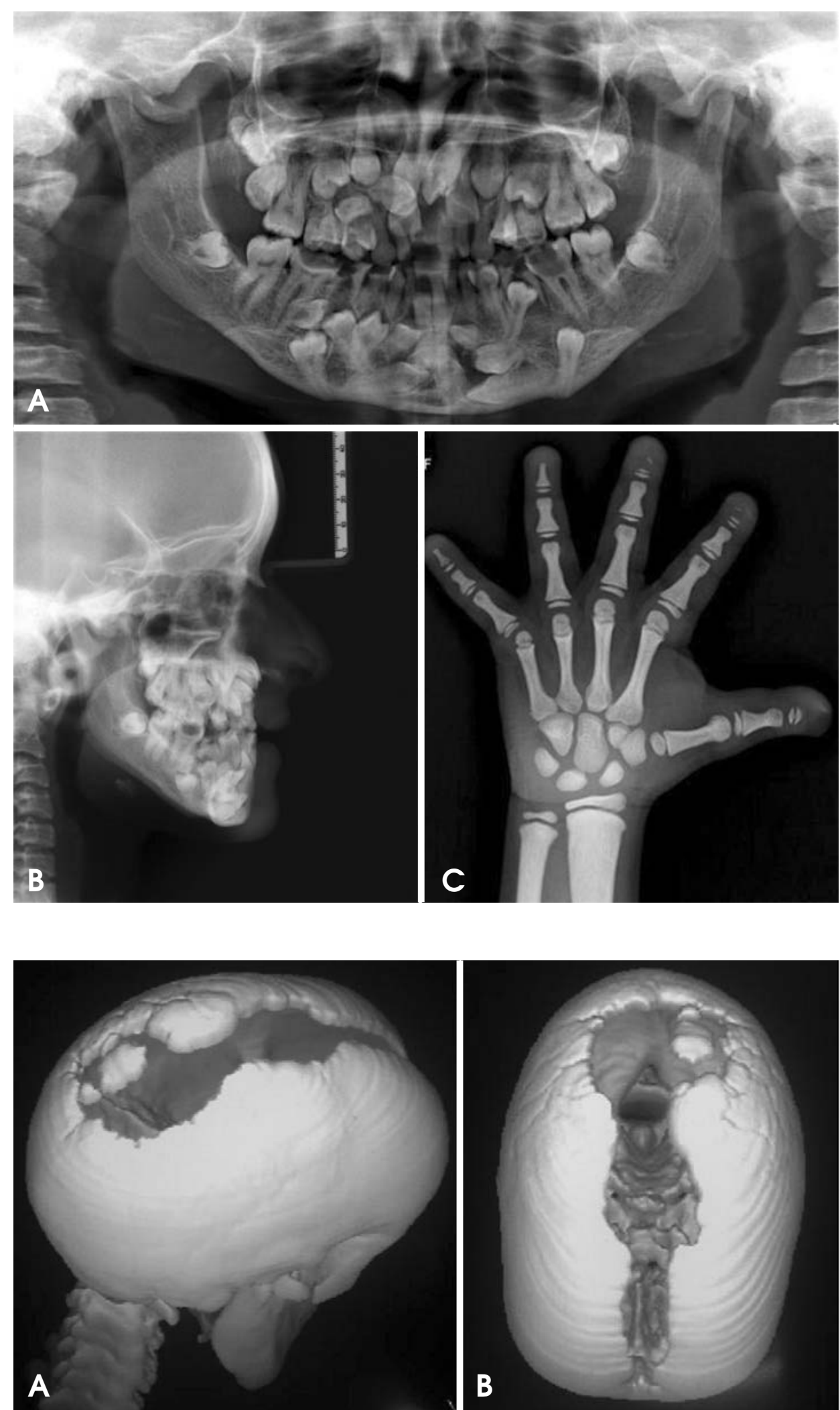

Fig. 3. A. Panoramic radiograph shows multiple impacted permanent and supernumerary teeth. B. Lateral cephalogram shows hypoplastic paranasal sinuses with relative mandibular prognathism. C. Hand-wrist radiograph shows acro-osteolysis of terminal phalanges.

Fig. 4. A and B. Three-dimensional CT images show open fontanelles and sutures, wormian bones in the lambdoidal region. hypoplasia, extensive dental caries, narrowing of pulp cavities, shortness of tooth root, hypodontia or supernumerary, and hypercementosis are specific to pyknodysostosis. ${ }^{2,4-6}$
Other reported abnormalities were midline antero-posterior ridge of the palate, high arched palate, macroglossia, and infection of unerupted permanent teeth follicles, lead- 
ing to abscess formation. Also, there were reports which observed anomalies of various permanent teeth. ${ }^{2,5,6}$

The intraoral features present were midline antero-posterior ridge of the palate, high arched palate along with persistence of deciduous teeth, crowding, enamel hypoplasia, and dental caries. Similar findings were reported by Fleming et al, ${ }^{2}$ Moniz et al, ${ }^{4}$ Hunt et al, ${ }^{5}$ and Schmitz et al. ${ }^{6}$

The radiological findings may show some degree of widening of the distal femur. The skull shows open anterior fontanelle and sutures with small facial bones, nonpneumatised paranasal sinuses, and flattened mandibular angle. Terminal phalanges of the hands are partially or totally aplastic with loss of ungual tufts. The acromial ends of the clavicles may be aplastic. Other abnormalities include failure of complete segmentation of the atlas, axis, and the lower lumbar spine, coxa valga and abnormal radioulnar articulation. $^{1,7}$

The radiological findings observed were hypoplastic paranasal sinuses with relative mandibular prognathism, acro-osteolysis of terminal phalanges, multiple impacted permanent and supernumerary teeth. Narrow left ramus, condyle and condylar notch, open fontanelles and sutures, and wormian bones in the lambdoidal region as revealed in 3D-CT image were also appreciable. The same features were present in the study by Maroteaux et $\mathrm{al}^{1}$ and Fleming et al. ${ }^{2}$

The histological appearance of bone is identical in osteopetrosis and pyknodysostosis, however in the latter disease there is a small and imperfect medullary canal along with attenuated Haversian canal system and microscopical evidence of haemopoiesis. ${ }^{3,8}$ Osteopetrosis is characterized by the endosteal production of bone with an apparent concomitant lack of physiologic bone resorption. Osteoblasts are prominent but osteoclasts are seldom found in significant numbers in tissue sections. The predominance of bone formation over resorption typically leads to the persistence of cartilaginous cores of bony trabeculae long after the replacement should have occurred in endochondral bones. Trabeculae themselves are disorderly in arrangement and the marrow tissue present is usually fibrous. ${ }^{10}$

MRI findings of pyknodysostosis generally reveal normal cortical thickness in the calvaria, however there is increased trabecular bone within the medullary cavity. There is limited literature describing the CT findings, which includes hypoplastic sinuses, poor dentition, and thickening of the calvaria. $^{5}$

The differential diagnosis of pyknodysostosis is established with osteopetrosis, cleidocranial dysplasia and idiopathic acro-osteolysis. In osteopetrosis, the bone marrow may be absent; it is therefore frequent for hematopoietic alterations to appear. Signs of compression of the cranial nerves exist such as facial paralysis, deafness, or pain. Cleidocranial dysplasia may seem like pyknodysostosis for presentation of agenesis or clavicular aplasia, as well as alterations of the skeletal bone membranes, however bone density is not increased. In idiopathic acro-osteolysis, the appearance of the patients is typical, with hypotelorism, exophthalmos, and an upturned nose. The angle of the mandible is acute and increased bone density is not present. ${ }^{9}$

The diagnosis of pyknodysostosis is primarily based on clinical features and radiographs; however a CTSK gene mutation analysis is the confirmatory test. ${ }^{3}$

There is no specific treatment as of date for this disorder and treatment is supportive. Since bone fractures are a primary threat, it is important that care is taken to prevent or minimize tendencies for a fracture to occur. ${ }^{3}$ Tooth extraction demands special care, such as carrying out the surgery as atraumatically as possible and with proper asepsis, due to the risk of fracture, especially in the mandible. ${ }^{9}$ However, bone healing is normal despite the fragility of bones. As a real complication, mandibular fracture and subsequent osteomyelitis should be considered when seemingly minor surgery such as tooth extraction or dental implants are indicated and osteomyelitis could become refractory due to osteosclerosis. Child patients should receive special dental care, particularly preventive treatment because of the group of mouth alterations described, in addition to periodic follow-up of their growth and craniofacial development. ${ }^{11}$ The prognosis of the disease is good and no more serious systemic alteration has been noted. Also, life expectancy for a pyknodysostosis patient is normal. ${ }^{3}$

\section{References}

1. Maroteaux P, Lamy M. Pyknodysostosis. Presse Med 1962; 70 : 999-1002.

2. Fleming KW, Barest G, Sakai O. Dental and facial bone abnormalities in pyknodysostosis: CT findings. AJNR Am J Neuroradiol 2007; $28:$ 132-4.

3. Mujawar Q, Naganoor R, Patil H, Thobbi AN, Ukkali S, Malagi N. Pyknodysostosis with unusual findings: a case report. Cases J 2009; 2 : 6544.

4. Moniz N, Queiroz EA, Freitas RR, Felix VB. Mandibular reconstruction with autogenous graft in patient presenting pyknodysostosis: case report. J Oral Maxillofac Surg 2006; 64 : 1292-5.

5. Hunt NP, Cunningham SJ, Adnan N, Harris M. The dental, craniofacial, and biochemical features of pyknodysostosis: a report of three new cases. J Oral Maxillofac Surg 1998; 56 : 497-504.

6. Schmitz JP, Gassmann CJ, Bauer AM, Smith BR. Mandibular 
reconstruction in a patient with pyknodysostosis. J Oral Maxillofac Surg 1996; 54 : 513-7.

7. Motyckova G, Fisher DE. Pycnodysostosis: role and regulation of cathepsin $\mathrm{K}$ in osteoclast function and human disease. Curr Mol Med 2002; 2 : 407-21.

8. Elmore SM. Pycnodysostosis: a review. J Bone Joint Surg 1967; $49: 153-62$.

9. Landa S, Esteban S, Montes E, Santamaria J, Vitoria A, Santo- laya JM. Maxillofacial alterations in a family with pycnodysostosis. Med Oral 2000; 5 : 169-76.

10. Rajendran R. Diseases of bone and joints. In: Rajendran R, Sivapathasundharam B. Shafer's textbook of oral pathology. 6th ed. Noida: Elsevier; 2010. p. 701.

11. Soares LF, Souza I, Cardoso AS, Pomarico L. Pyknodysostosis: oral findings and differential diagnosis. J Indian Soc Pedod Prev Dent 2008; 26 : S23-5. 